\title{
Integration of Deformation Processing with Additive Manufacture of Ti-6Al-4V Components for Improved Grain Structure and Texture \\ DOI:
}

10.1002/9781119093466.ch55

\section{Document Version}

Accepted author manuscript

Link to publication record in Manchester Research Explorer

Citation for published version (APA):

Prangnell, P., Donoghue, J., Jagjit, S., \& Wescott, A. (2015). Integration of Deformation Processing with Additive Manufacture of Ti-6Al-4V Components for Improved Grain Structure and Texture. 437. Paper presented at TMS 2015 A146th Annual Meeting and Exhibition, Orlando, United States. https://doi.org/10.1002/9781119093466.ch55

\section{Citing this paper}

Please note that where the full-text provided on Manchester Research Explorer is the Author Accepted Manuscript or Proof version this may differ from the final Published version. If citing, it is advised that you check and use the publisher's definitive version.

\section{General rights}

Copyright and moral rights for the publications made accessible in the Research Explorer are retained by the authors and/or other copyright owners and it is a condition of accessing publications that users recognise and abide by the legal requirements associated with these rights.

\section{Takedown policy}

If you believe that this document breaches copyright please refer to the University of Manchester's Takedown Procedures [http://man.ac.uk/04Y6Bo] or contact uml.scholarlycommunications@manchester.ac.uk providing relevant details, so we can investigate your claim.

\section{OPEN ACCESS}




\title{
Integration of Deformation Processing with Additive Manufacture of Ti-6Al- 4V Components for Improved $\beta$ Grain Structure and Texture
}

\author{
Jack Donoghue ${ }^{1}$, Jagjit Sidhu ${ }^{2}$, Andrew Wescott ${ }^{2}$, and Phillip Prangnell ${ }^{1}$ \\ ${ }^{1}$ Materials Science Centre, University of Manchester, Grosvenor St. Manchester M13 9PL, UK \\ ${ }^{2}$ BAE Systems, Sowerby Research Centre, PO Box 5, Filton, Bristol BS12 7QW, UK
}

Keywords: Additive Manufacture, Deformation Processing, Microstructural Refinement

\begin{abstract}
With Ti alloys like Ti-6Al-4V, the solidification conditions across virtually all AM platforms lead to strongly textured, coarse columnar, $\beta$ grain structures. Transformation to $\alpha$ on cooling dilutes the texture, but significant texture is still inherited which contributes to undesirable anisotropy in AM parts. In the work presented a deformation step has been integrated into the manufacture of components produced by the blown powder method, using an Ultrasonic Impact Treatment (UIT), which has the additional benefit of reducing residual stresses. It has been found that the introduction of surface deformation to each layer can lead to a greatly refined grain structure with a more randomised texture. To investigate the origin of this effect, reconstruction of the $\beta$ grain structure and texture from the $\alpha$ EBSD measurements has been used to characterise the high temperature $\beta$ microstructure.
\end{abstract}

\section{Introduction}

There is potential for the aerospace industry to greatly benefit from Additive Manufacture (AM) as it allows the near-net-shape manufacture of components [1-7] and provides more design freedom than traditional manufacturing, which can facilitate substantial weight savings through better design optimisation [1]. Ti-6Al-4V is one of the most widely used titanium aerospace alloys due to its high specific properties [8]. However, optimum performance is traditionally achieved with this material through the thermomechanical processing (TMP) [9]. In contrast, $\mathrm{AM}$ is based on the layerwise deposition of material through melting and this can lead to quite different microstructures and textures $[2,3]$.

Of particular concern in aerospace components with alloys like Ti-6Al-4V is that coarsecolumnar primary $\beta$-grain structures are nearly always observed in AM processes [2,3,7]. Transformation to $\alpha$ on cooling refines the microstructure, however, significant texture is still inherited in the $\alpha$ phase and this can contribute to anisotropy. A coarse $\beta$ structure develops in AM because there is a steep thermal gradient in the melt pool ahead of the solidification front. When combined with the high partition coefficients of $\mathrm{Al}$ and $\mathrm{V}$ in Ti [10], this limits the degree of constitutional supercooling that is possible and nucleation ahead of the solidification front becomes very difficult. As a result, homo-epitaxial re-growth takes place within each melted layer, allowing coarse directional grain structures to develop that can grow up through many deposited layers. [2,3,7]. In addition, because of the preferred $\langle 001\rangle$ growth direction in cubic metals, the large columnar grains tend to have a strong $\beta<001>$ fibre texture parallel to the average solidification direction within a particular AM process [2,3]. Disrupting this columnar structure by metallurgical means is challenging as there are few options for grain refining additions in titanium [11]. 
In $\alpha-\beta$ alloys like Ti-6Al-4V, upon rapid cooling below the $\beta$ transus, the majority of the $\beta$ phase transforms to fine Widmanstätten $\alpha$ lamellae [9] while the remainder of the parent $\beta$ is retained as thin layers between the $\alpha$ plates [12]. The texture is weakened by the transformation as there are 12 possible variants of the $\alpha$ phase formed within each $\beta$ grain, as described by the Burgers orientation relationship [13]; $\{110\}_{\beta}\left\|\{0002\}_{\alpha},\langle 111\rangle_{\beta}\right\|\langle 11 \overline{2} 0\rangle_{\alpha}$. However, on reheating back to the $\beta$ phase field, which in AM generally occurs several times within each deposited layer [3], the retained $\beta$ phase re-grows with its original parent orientation, consuming $\alpha$, and thus thermal cycling does not lead to any significant texture weakening.

In AM processes a component is built up from multiple tracks with a small moving heat source which can lead to the development of substantial residual stresses and distortion [5]. Similar stresses are accrued with a single track deposition in traditional welds, and have previously been relieved by imparting a compensating plastic strain by treatments such as peening [14] and rolling [15]. However, when a rolling step was introduced into a wire-plasma AM process, in addition to a decrease in residual stresses, a large reduction in the prior $\beta$ grain size was observed accompanied by a weakening of the $\beta<100>$ fibre texture [16]. Given the relatively small plastic strains applied in this work, this is quite a surprising result and the mechanism of grain refinement still remains to be fully explored. In the present work, the laser blown powder technique (LBP) has been integrated with Ultrasonic Impact Treatment (UIT) (a form of peening [17]), of the deposited layers to determine if a similar microstructural improvement could be achieved with this higher layer resolution AM process.

\section{Experimental}

\section{$\underline{\text { AM Builds }}$}

Simple linear $\sim 250$ long, $\sim 15$ wide, and $\sim 65 \mathrm{~mm}$ high walls were built up using a laser blown powder AM system with gas atomised Ti-6Al-4V powder. The power of the Nd-YAG Trumph laser used was $1000 \mathrm{~W}$ and the deposits were produced with a constant travel speed of $15 \mathrm{~mm} / \mathrm{s}$. The powder was blown into the melt pool by an argon carrier gas and oxidation was prevented by containment within an argon filled environment. Deposition occurred on a base plate of similar composition to the powder. 20 tracks of material were deposited to make up each layer and 75 layers were deposited to make each wall, with a layer height of $\sim 0.9 \mathrm{~mm}$. The build sequence for each layer was identical, but the raster direction was reversed. Thus below, $x$ is taken to be the direction parallel to the length of the wall and beam travel direction and $z$ is normal to the layers. Three builds were made for comparison purposes; i) a standard build with no deformation, ii) with an Ultrasonic Impact Treatment (UIT) applied every 5 layers, and iii) with UIT applied after every deposited layer. UIT was applied when the material had cooled down uniformly across the top surface of each layer.

Characterization and $\beta$ - Phase reconstruction

For metallographic examination the walls were sectioned in the $x-z$ and $y-z$ planes, before being ground and polished. Samples were etched with Kroll's reagent for optical microscopy. Orientation and texture analysis was carried out by Electron Back Scatter Diffraction (EBSD) in a Camscan FEG-SEM. Orientation maps were generated with an Oxford instruments EBSD system, operating Channel 5 software. The textures of the reconstructed high temperature $\beta$ phase (see below) and room temperature $\alpha$ phase are depicted by pole figures. As the $\langle 100\rangle$ fibre texture was aligned with the columnar grain growth direction, and this was found not to be coincident with the $y$, and $z$ directions in the build geometry. A second reference frame has been used where $x, y$, and $z$ have been rotated around the $x$ axis to $x, y^{\prime}$, and $z$ ' so that $z$ ' is aligned 
with the dominant grain growth direction, or $\langle 100\rangle$ fibre axis. This procedure has been adopted throughout to produce the IPF coloured orientation maps depicted below (See figure 1).

Because EBSD measurement are made at room temperature, where it is very difficult to resolve the small quantity of retained $\beta$ phase, reliable orientation date could only be obtained from the $\alpha$ phase. Thus, in order to characterise the primary $\beta$-phase, a reconstruction procedure was applied (developed at The University of Sheffield and described elsewhere [18]) which calculates the most probable parent $\beta$ orientation for any $\alpha$ grain by utilising the Burgers relationship, and comparison of the misorientation between neighbouring $\alpha$ plate variants.

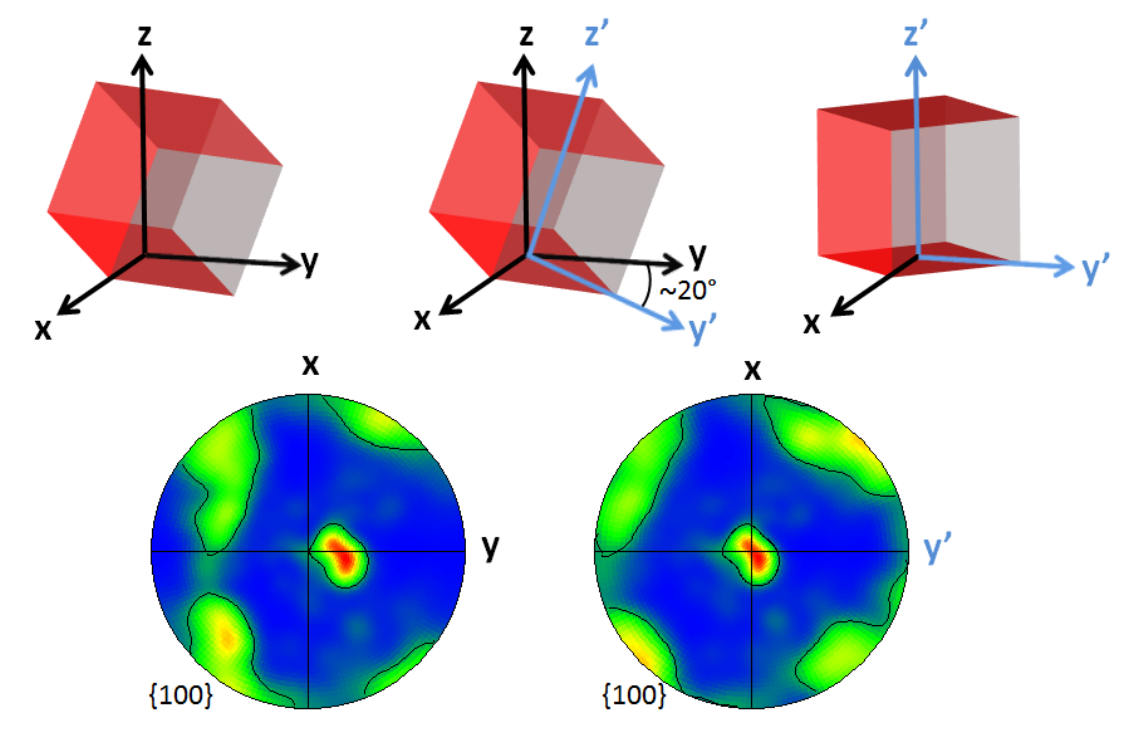

Figure 1. Example of the rotation typically required of $\sim 20^{\circ}$ around the $x$ axis of the build geometry $(x, y, z)$ to $\left(x, y^{\prime}, z^{\prime}\right)$ to align $z$ ' with the dominant $<001>$ fibre axis of the columnar grain growth direction for the $\beta$ phase.

\section{Results and Discussion}

Optical images of the conventional LBP build (i) can be seen in figure 2 where it is possible to readily distinguish the coarse columnar prior- $\beta$ microstructure as well as microstructural banding. Similar banding has been observed in other AM processes $[4,19]$ and is associated with microstructural changes that occur across the $\beta$ transus [4]. Epitaxial-growth of the prior $\beta$ columnar grains up through many deposited layers can clearly be observed in figure $2 \mathrm{a}$, where the grains are approximately $\sim 0.25 \mathrm{~mm}$ wide and over $1 \mathrm{~cm}$ in height, some being taller than the field of view. The 'fish-scale' banding morphology in figure $2 \mathrm{a}$ is related to the semi-circular cross-section of the weld pool seen when viewed transverse to the direction of travel, $(y-z$ plane $)$, which is tilted due to overlap with the previously deposited neighbouring track. In comparison, the horizontal banding observed in figure $2 \mathrm{~b}$ occurs due to the travel of the heat source which, when viewed at steady state in this plane, maintains a constant depth for the isotherm that reaches the $\beta$ transus temperature.

Also visible in this cross-section is curvature of the grains towards the $y$ direction, caused by them following the maximum thermal gradient at the solidification front from the tilted bowlshaped melt pool. In comparison the tilt of the grains is very slight in figure $2 b$, because from this perspective the rapid movement of the laser in $x$ elongates the melt pool causing a flatter 
solidification front. In addition, tilt in $x$ is effectively suppressed by the reversal of the beam travel direction with each successive pass which results in the average preferred growth direction being parallel to $z$ [3]. In contrast tilt in $y$ in the $y$-z plane is reinforced in each successive layer, as regardless of the direction of deposition in $x$, the tracks were stepped across from the same side of the build and thus the curvature of the solidification front was always the same when viewed in this plane.

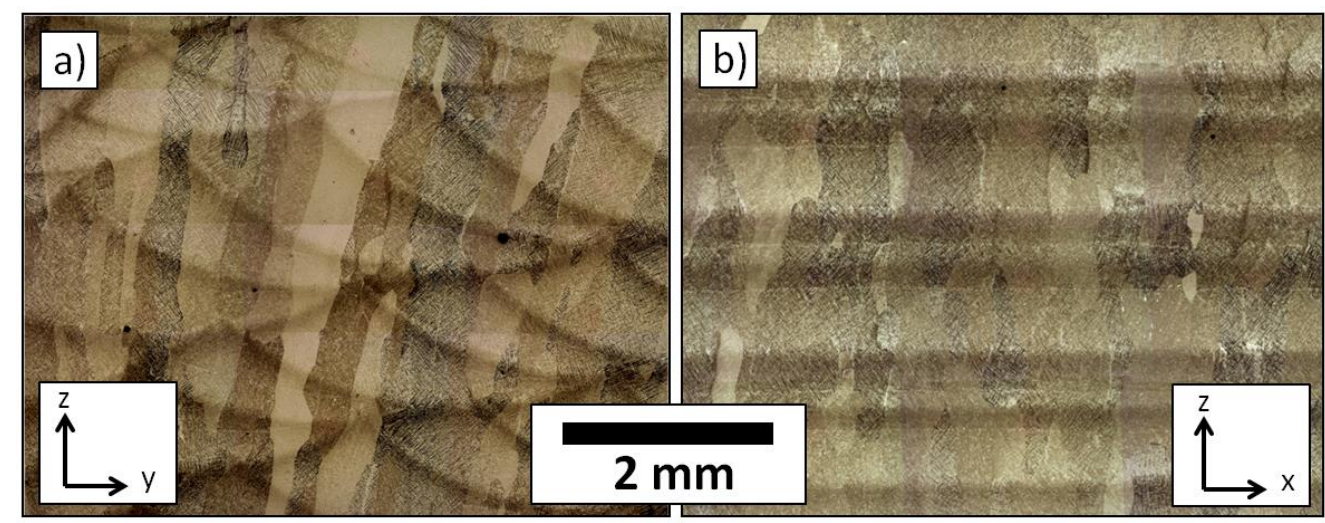

Figure 2. Etched microstructures of the standard wall (no UIT) in the $z-y(\mathrm{a})$, and $z-x(\mathrm{~b})$ planes.

Effect of UIT on the primary $\beta$ grain structure

In figure 3 the primary $\beta$ grain structures are clearly seen in IPF orientation maps reconstructed from EBSD measurements of the $\alpha$ orientations present at room temperature. Figure 3 includes maps of the three samples produced with and without the UIT treatment, where the data has been rotated as described in figure 1 to align $z^{\prime}$ with the principle $<001>$ fibre direction, which is coloured red.
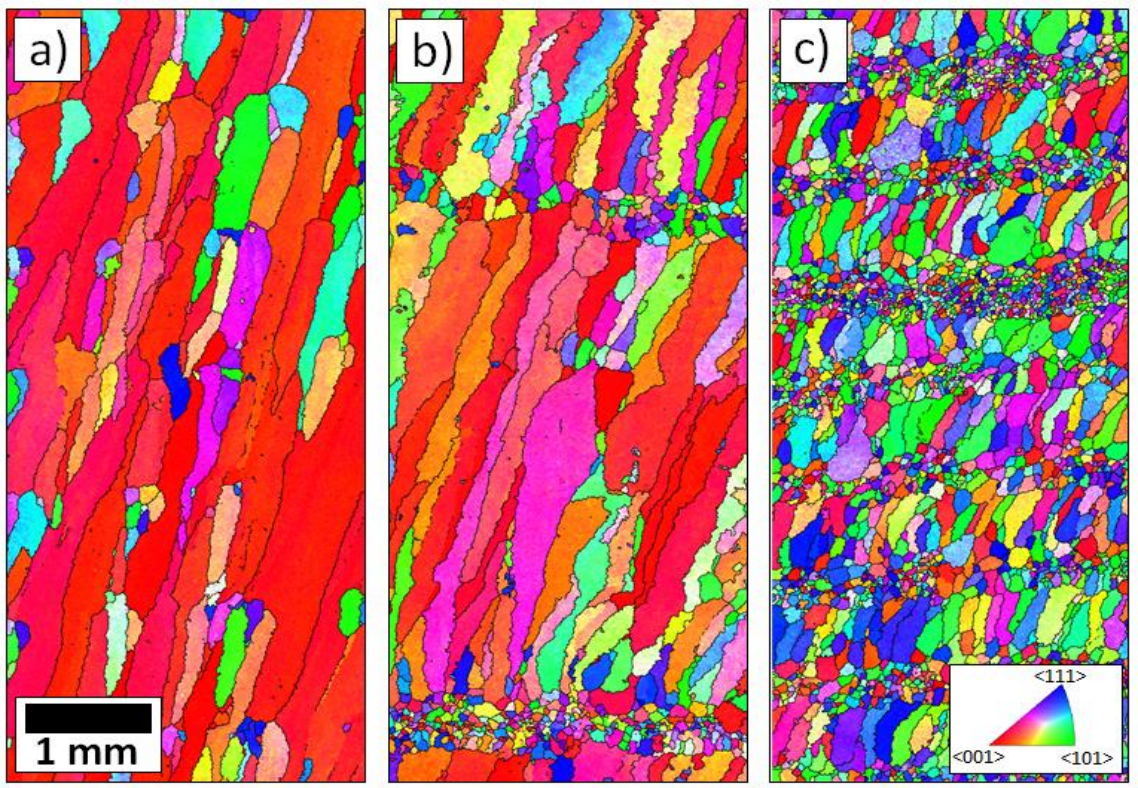

Figure 3. Reconstructed EBSD maps of the $y-z$ plane from (a), the untreated build, (b) with UIT applied every 5 layers and (c), UIT applied every layer. 
In the untreated control sample, shown in figure 3a, large columnar grains are again clearly visible and it is evident from their dominant red colour that the grains have a strong <100> preferred growth direction. In the second sample shown in figure $3 \mathrm{~b}$ the UIT treatment was applied to every fifth deposited layer, and as a result a band of finer equiaxed grains can be observed, breaking up the columnar structure and limiting the height of each columnar band to $\sim 4 \mathrm{~mm}$. The narrow bands of equiaxed grains that have been produced within each deformed layer are much finer than the columnar grains, $(\sim 50 \mu \mathrm{m}$ in diameter $)$ and have more random orientations. However, too few grains have been sampled to give reliable statistics. In figure $3 \mathrm{c}$ the effect of applying the UIT to every layer is also shown, which leads to an alternating structure of fine equiaxed grains and smaller columnar grains. The more frequent application of UIT now limits the columnar grains to a height of only $\sim 0.5 \mathrm{~mm}$, leading to a more random texture.

\section{Effect of UIT on texture in the LBP process}

As can be seen from the $\beta<001>$ pole figure in figure $4 \mathrm{a}$, from the standard build without the UIT treatment, there is strong $<100\rangle$ alignment along the columnar grain direction. This confirms that the moving melt pool solidification conditions, combined with layer-by-layer deposition, leads to epitaxial growth of a coarse, preferentially aligned $\beta$ grain structure in a Ti6Al-4V alloy. However, although there are statistical concerns as only a relatively small number of grains were sampled $(\sim 500)$, rather than forming a true $\langle 100\rangle$ fibre as has been reported in the literature [20], it appears that the columnar grains are predominantly spread around a cube component that is rotated $\sim 45^{\circ}$ around $z^{\prime}$, relative to the laser travel direction. It should also be noted that, as shown in figure 1, the fibre axis was originally tilted around $x$ by $20^{\circ}$ away from the layer normal direction $(z)$ because of the curvature of the melt pool base and the effect track overlap had on the tilt of the melt pool surface. Cube [3] and $45^{\circ}$ rotated cube [2] components have been previously reported in AM builds using an electron beam method [2].

A possible explanation for the cube orientation has been given by Antonysamy et al. whom suggested that a repeated alternating orthogonal raster pattern can encourage alignment of the <100> growth direction with the beam path as well as in the build direction [3]. However, this explanation is not applicable here as the cube texture is orientated at $45^{\circ}$ to the travel direction. This behaviour needs further investigation, but in the LBP process it is possible that it is related to the shape of the melt pool where an elliptical, or tear drop shape, would cause grain growth in the $x-y$ plane to occur predominately at an angle relative to the beam travel direction.
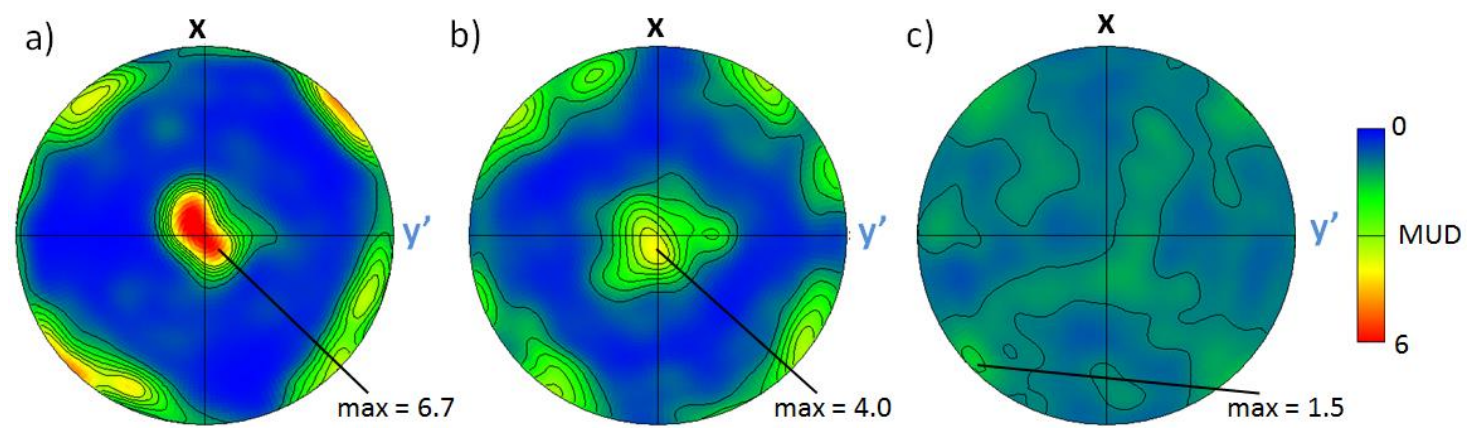

Figure 4. $<100>$ pole figures indicating the $\beta$ texture strength for (a) the standard untreated build, (b) LBP with UIT applied every 5 layers, and (c) LBP with UIT applied to every layer.

In figure $4 \mathrm{~b}$ it can be seen that when UIT was applied to every fifth layer the average texture was substantially weakened by a factor of $\sim 40 \%$. As the layers of fine randomly orientated grains 
produced by this treatment were very thin and did not contribute greatly to the sample volume. The texture weakening has occurred largely as a result of the bands of columnar grains not being able to develop such strong preferred orientations, owing to their growth being repeatedly cut-off by the application of the deformation treatment; i.e. the reduction in texture strength is more related to the length of stable columnar grain growth that is needed to re-establish a strong $<100>$ texture after it is interrupted.

This interpretation can be better demonstrated by separating the textures obtained from the two grain structures, as has been done in figure 5. From these results it can be seen that the main $<001>/ / z^{\prime}$ component taken from the columnar band data has only a slightly stronger maximum intensity than that seen for the whole volume averaged texture (figure $4 \mathrm{~b}$ ). In addition, it can now be seen that the thin band of refined grains has a very weak texture, which still contains some of the same $45^{\circ}$ rotated cube component.
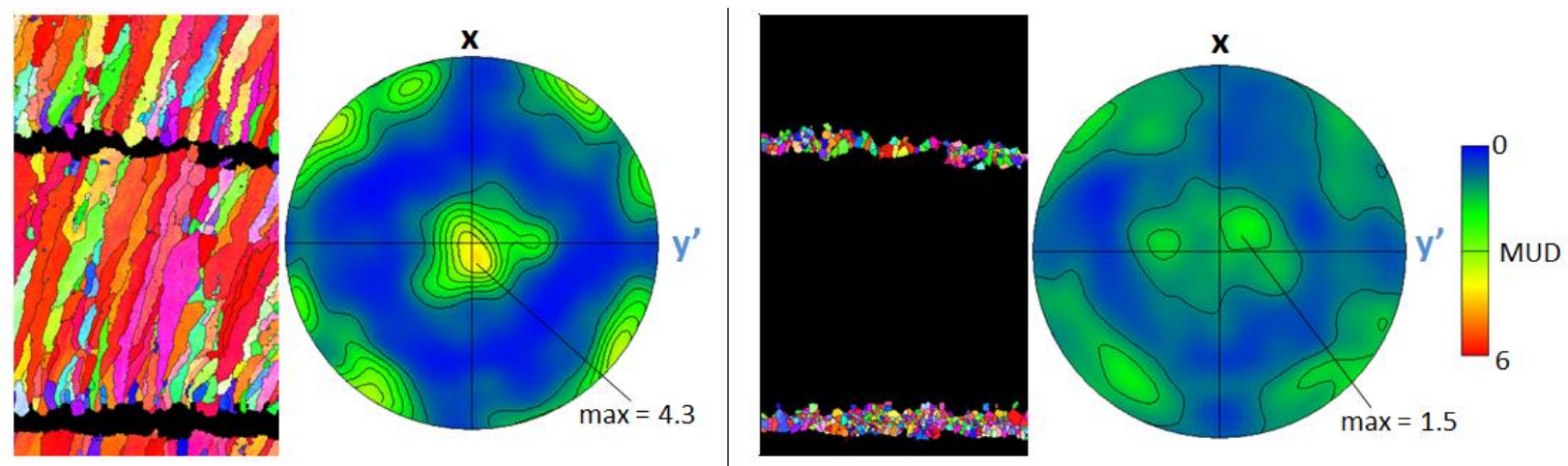

Figure 5. Separation of the textures of the sample deformed every five layers into their columnar and refined regions.

More important than the above results, in terms of understanding the potential for the ultrasonic impact treatment to improve texture in AM, is that when UIT was applied to every deposited layer the $\beta$ texture became very weak (figure $4 c$ ). In fact, from figure $4 \mathrm{c}$ it can be seen that when UIT was used repeatedly on every layer the resultant texture of the wall was effectively random.
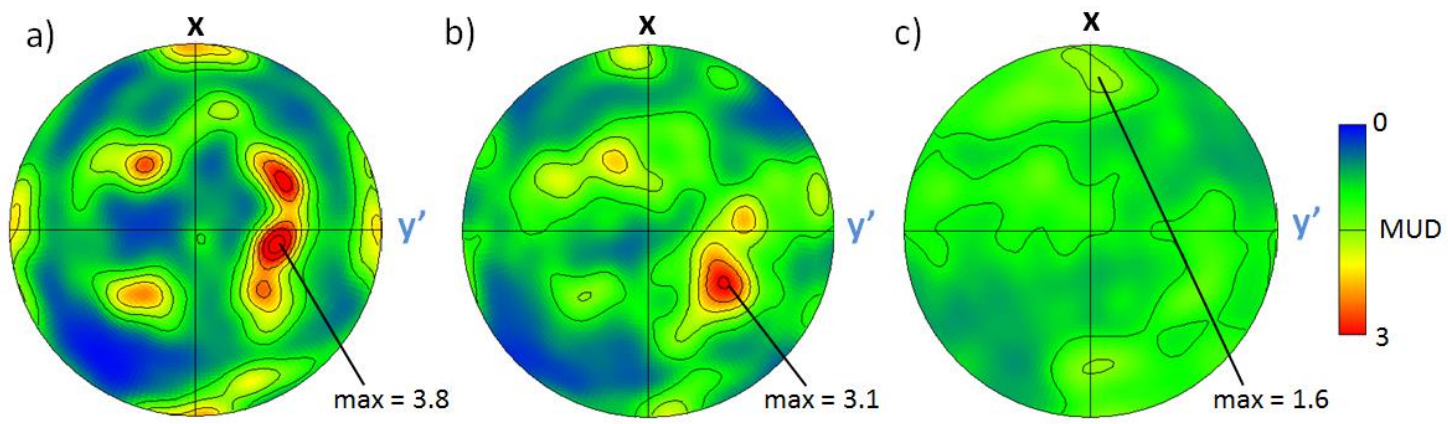

Figure 6. $\{0002\}$ basal pole figures indicating the room temperature $\alpha$ texture strength of (a) the standard build, (b) with UIT applied every 5 layers and (c) the texture when UIT was applied every layer.

As described in the introduction, owing to the 12 orientations possible from the Burgers relationship, transformation to $\alpha$ at room temperature significantly weakened all the final textures 
of the AM parts relative to those seen for the primary $\beta$ grain structures that developed during solidification (figure 6 ). Thus, the strong $\beta$ texture seen in the standard wall transformed to a stronger $\alpha$ texture than in the ultrasonic impact treated walls, but in both cases the texture was weakened by the transformation. In contrast, because of the original weakened $\beta$ texture, in the build that was treated with the UIT process every layer the $\alpha$ texture was effectively eliminated.

Origin of the microstructural refinement

In AM, the effect of the deformation applied to the top surface of a build by the UIT process cannot be interpreted simply as 'recrystallisation' because of the complex thermal cycle the material experiences and the fact that there is a massive $\alpha \rightarrow \beta$ phase transformation that occurs on re-heating each layer above the $\beta$ transus temperature, by subsequent passes of the heat source. In the undeformed standard sample establishment of a columnar microstructure is enabled by the retained $\beta$ in the room temperature transformation microstructure, which acts as nuclei for the primary $\beta$ microstructure to re-grow upon re-heating above the $\beta$ transus. On solidification of a new added layer, epitaxial re-growth then occurs from this re-created $\beta$ grain structure. It is therefore apparent that deformation of the room temperature microstructure by UIT must result in the development of new $\beta$ orientations within the retained $\beta$ phase that then can grow as new grains on reheating in the next pass. Alternatively, intense deformation of the $\alpha$ lamellae, and possibly by processes like twinning, could cause new $\beta$ orientations to nucleate within the $\alpha$ phase during reheating.

The UIT process itself only introduces local deformation below the surface with a diminishing strain with depth, and it is evident most of this region is lost by re-melting as the next layer is deposited. With the current process parameters the UIT treatment is thus only just successful, because the depth of deformation is just deep enough to survive application of the next layer and provide sufficient deformation to induce refinement of the $\beta$ phase when it re-grows; i.e. we do not see a graduated microstructure across the refined zones to correspond with the decrease in stored energy with depth, that would be expected from the UIT process. Instead only a thin layer of refined grains is observed with a distinct boundary between the columnar structure underneath, implying that there must be a critical condition to overcome in order for the grain refinement to occur. As in the $y-z$ plane the refined zones occur in horizontal bands parallel to the deformed surface, rather than following the fish-scale banding pattern that indicates the temperature profile below each melt track, it can be deduced that this critical condition is strain limited. This refined layer of random $\beta$ grain orientations then goes on to disrupt the development of columnar growth of the same grain orientations up through many layers. When allowed to continue, this behaviour would normally result in the growth of very large grains that are progressively selected to be more closely aligned with the optimum growth direction and, hence, the UIT treatment also disrupts the development of the strong <001> texture seen in the standard build.

\section{Conclusions}

- As has been noted in other AM processes, a coarse columnar prior $\beta$ grain structure with a strong texture is formed by preferential $\langle 100\rangle$ growth in the standard LBP process. However, a rotated cube component has been observed rather than a $<100>$ fibre texture.

- The addition of UIT to every layer is a very effective process step for interrupting columnar growth of the coarse $\beta$ grain structure normally seen on solidification. This is achieved by producing a band of finer equiaxed prior $\beta$ grains with a randomised texture.

- Although the depth of surface deformation obtained by UIT in this study was not great enough to fully refine the microstructure of each layer, this could be achieved with an 
AM technique that has a thinner layer height and/or by a deformation process that can impart a greater depth of deformation.

\section{Acknowledgements}

The authors would like to thank Dr. B. Wynne (Sheffield) for provision of the $\beta$ reconstruction software. J. Donoghue is grateful for financial support provided by LATEST2 (EP/G022402/1) and Airbus, UK. We are also grateful to Prof. S. Williams group at Cranfield for proposing the idea of applying deformation to AM processing and the EPSRC CIM in Laser Based Production Processes (EP/K030884/1).

\section{References}

1. P. Kobryn, N. Ontko, L. Perkins, and J. Tiley, (2006).

2. S. S. Al-Bermani, M. L. Blackmore, W. Zhang, and I. Todd, Metall. Mater. Trans. A 41, 3422 (2010).

3. A. A. Antonysamy, J. Meyer, and P. B. Prangnell, Mater. Charact. (2013).

4. F. Wang, S. Williams, P. Colegrove, and A. a. Antonysamy, Metall. Mater. Trans. A 44, 968 (2012).

5. P. Colegrove, H. E. Coules, J. Fairman, F. Martina, T. Kashoob, H. Mamash, and L. D. Cozzolino, J. Mater. Process. Technol. 213, 1782 (2013).

6. K. Taminger and R. Hafley, NATO AVT (2006).

7. S. Reginster, A. Mertens, H. Paydas, J. Tchuindjang, Q. Contrepois, T. Dormal, O. Lemaire, and J. Lecomte-Beckers, Mater. Sci. Forum 765, 413 (2013).

8. R. R. Boyer, Mater. Sci. Eng. A 213, 103 (1996).

9. G. Lütjering, Mater. Sci. Eng. A 243, 32 (1998).

10. M. J. Bermingham, S. D. McDonald, M. S. Dargusch, and D. H. StJohn, J. Mater. Res. 23, 97 (2011).

11. M. J. Bermingham, S. D. McDonald, K. Nogita, D. H. St. John, and M. S. Dargusch, Scr. Mater. 59, 538 (2008).

12. G. Lütjering and J. C.Williams, Titanium, 2nd ed. (Springer, Berlin, n.d.).

13. W. G. Burgers, Phys. I 561 (1933).

14. L. Hacini, N. Lê, and P. Bocher, Exp. Mech. 49, 775 (2008).

15. D. F. O. Braga, H. E. Coules, T. Pirling, V. Richter-Trummer, P. Colegrove, and P. M. S. T. de Castro, J. Mater. Process. Technol. 213, 2323 (2013).

16. A. A. Antonysamy, Microstructure, Texture and Mechanical Property Evolution during Additive Manufacturing of Ti6Al4V Alloy for Aerospace Applications, University of

Manchester, 2012.

17. S. Roy and J. Fisher, Int. J. Steel Struct. (2005).

18. P. S. Davies, An Investigation of Microstructure and Texture Evolution in the Near-A Titanium Alloy Timetal 834, University of Sheffield, 2009.

19. E. Brandl, A. Schoberth, and C. Leyens, Mater. Sci. Eng. A 532, 295 (2012).

20. P. Kobryn and S. Semiatin, J. Mater. Process. Technol. 135, 330 (2003). 